OPEN ACCESS

Edited by:

Hongbing Ding,

Tianjin University, China

Reviewed by: Mohammad Ali Faghih Aliabadi, Shahid Beheshti University, Iran Jiang Bian,

China University of Petroleum (Eas China), China

${ }^{*}$ Correspondence: Z. Zhou zznwt@163.com P. Wiśniewsk piotr.wisniewski@polsl.pl

Specialty section: This article was submitted to Process and Energy Systems Engineering, a section of the journal Frontiers in Energy Research

Received: 20 November 2021 Accepted: 13 December 2021 Published: 06 January 2022

Citation:

Liu C, Li Y, Zhou Z and Wiśniewski $P$ (2022) Effect of Cascade Surface Roughness on Boundary Layer Flow Under Variable Conditions. Front. Energy Res. 9:818828. doi: 10.3389/fenrg.2021.818828

\section{Effect of Cascade Surface Roughness on Boundary Layer Flow Under Variable Conditions}

\author{
C. $\mathrm{Liu}^{1,2}, \mathrm{Y}^{\mathrm{Li}} \mathrm{i}^{1}$, Z. Zhou ${ }^{1 \star}$ and P. Wiśniewski ${ }^{3 *}$
}

${ }^{1}$ School of Electrical and Power Engineering, China University of Mining and Technology, Xuzhou, China, ${ }^{2}$ Xuhai College, China University of Mining and Technology, Xuzhou, China, ${ }^{3}$ Department of Power Engineering and Turbomachinery, Silesian University of Technology, Gliwice, Poland

Under the influence of many factors, the surface roughness of the cascade will change during turbomachinery operation, which will affect the boundary layer flow of the cascade. In this article, the effects of cascade surface roughness on boundary layer flow under variable conditions are analyzed by experiments and numerical simulation. The results show that with the increase of roughness, the total pressure loss coefficient of the cascade decreases first and then increases. The larger the Reynolds number is, the greater the total pressure loss coefficient is, and the sensitive area of loss change is changed. In the sensitive area, the roughness has a greater influence on cascade loss. There are separation bubbles at the suction front edge of smooth cascades. With the increase of roughness, the degree of turbulence increases, and the transition process is accelerated. When the roughness is between 74 and $150 \mu \mathrm{m}$, the separation bubble disappears and the separation loss decreases. In conclusion, the aerodynamic loss of the cascade increases with the increase of roughness, and the cascade efficiency decreases. However, roughness can restrain the flow separation and reduce the separation loss. The two have gone through a process of one and the other. When the roughness is $74 \mu \mathrm{m}$, the displacement thickness, momentum thickness, and shape factor at the back of the cascade are the minimum.

Keywords: roughness, boundary layer, flow separation, cascade, momentum thickness

\section{INTRODUCTION}

Turbomachinery is an important device for energy conversion. In the process of working, the blade cascade is affected by many factors such as working conditions, wear, and corrosion, leading to the change of blade surface roughness. Therefore, roughness, as a passive control method (Tani and Hama, 1953), can eliminate the adverse effects of flow separation on cascade performance, improve the efficiency of turbomachinery, and maintain turbomachinery stability.

Khalfallah and Koliub (2007) studied the influence of dust on the performance of wind turbines, pointing out that surface roughness occurs in the manufacturing process of wind turbine blades. Tarabrin et al. (1998a) numerically calculated the arrival rate of particles to the blade surface and found that diffusion was the most important factor for deposition on the suction surface. Tarabrin et al. (1998b) found that blade scaling would cause aerodynamic performance deterioration of axial flow compressors. 
TABLE 1 | Cascade parameters.

\begin{tabular}{lc}
\hline Design parameter & Value \\
\hline Chord length $C /(\mathrm{mm})$ & 93.79 \\
Cascade spacing $S /(\mathrm{mm})$ & 81.25 \\
Blade height $\mathrm{H} /(\mathrm{mm})$ & 100 \\
Inlet mental angle $\beta_{1} /\left(^{\circ}\right)$ & 90 \\
Incidence $i /\left(^{\circ}\right)$ & 15 \\
Outlet mental angle $\beta_{2} /\left(^{\circ}\right)$ & 76.9 \\
Airflow turning angle $\theta /\left(^{\circ}\right)$ & 13.1 \\
Cascade solidity & 1.154
\end{tabular}

Ishida et al. (2001) glued 40-mesh sand paper to the end wall of the centrifugal blower, which inhibited the flow separation of the blower blade and reduced the outflow loss. Meanwhile, roughness increased the kinetic energy loss caused by friction and reduced the pressure of the blower by about $1 \%$, ultimately improving its stall margin. Boese and Fottner (2002) applied the groove membrane to a high load compressor cascade to control its suction surface separation. After optimizing the flow of the groove structure, the total pressure loss coefficient of the compressor decreased by nearly $5 \%$. Gbadebo et al. (2004) found through numerical simulation and experimental research that for the single-stage static chord length, the roughness at the leading edge could cause a sharp decline in performance. Koch and Smith (1980) analyzed the boundary layer characteristics caused by surface roughness changes caused by scaling on axial flow compressors through experimental studies.

Due to the diversity of cascade operating environments, studies on cascade roughness are often coupled with operating conditions, and the relationship between the effect of roughness and incoming flow Reynolds number is a very important research issue. Schreiber et al. (2002) studied the changes of roughness in the Reynolds number range of $7 \times 10^{5}$ to $3 \times 10^{6}$ on compressor CDA blade performance. Back et al. (2012) studied the influence of roughness on the performance of low-speed compressor cascades under different Reynolds numbers of inlet flow and expounded the relationship between critical roughness and critical Reynolds number. Scha"ffler (1980) experimentally studied boundary layer characteristics of roughness blades of axial flow compressors at different Reynolds numbers. Leipold et al. (2000) measured the loss and boundary layer parameters of high-load transonic compressor cascades under the influence of roughness at different Reynolds numbers of incoming flow and analyzed the effect of surface roughness on inhibiting boundary layer separation at low Reynolds numbers.

Due to different design parameters and operating conditions of cascades, many relevant criteria of roughness influence are not universal, and the existing conclusions are not completely consistent. In order to understand the relationship between the roughness effect and incoming Reynolds number, it is necessary to conduct an in-depth study on the effect of surface roughness and incoming Reynolds number on the cascade boundary layer. In conclusion, roughness has both advantages and disadvantages on the cascade. Therefore, it is an important topic to study the effect of roughness on cascade boundary layer flow (Shamsoddin and Porté-Agel, 2017) under variable working conditions. In this article, the effects of roughness on cascade boundary layer flow under different Reynolds numbers are studied by numerical simulation and experimental verification, which provides a basis for flow separation control by roughness.

\section{RESEARCH CONTENTS AND METHODS}

In this article, it takes an NACA-65 airfoil model as the research object. The basic geometric parameters and their values are shown in Table 1.

The O-H grid was used to capture the flow state near the wall, and the position of the blade surface and front and back edges were grid-encrypted. Meshing ensures that $y+$ is less than 1 , and the cascade mesh is shown in Figure 1.

Mesh independence validation is shown in Figure 2. It can be observed that when the number of cells reaches $1.5 \times 10^{6}$, the static pressure coefficient does not change when the number of cells continues to increase. Therefore, selecting the number of cells as $1.5 \times 10^{6}$ for calculations can ensure the mesh independence.

CFX is employed to solve the three-dimensional steady-state flow over the cascade. For flow separation and transition phenomena on the cascade surface, shear-stress transport (SST) $\mathrm{k}-\omega$ formulation coupled with the $\gamma-R e_{\theta}$ transition model is selected for numerical calculations, and the roughness correction term $k$ is added to the transition model.

At present, the universal parameter for measuring surface roughness is the equivalent sand roughness $k_{s}$ proposed by Nikuradse (1950), Schlichting (1979). Nikuradse proposed the dimensionless roughness parameter $k^{+}$. To facilitate the calculation of $k^{+}$, the formula is arranged as follows:

$$
\begin{aligned}
k^{+} & =\operatorname{Re}_{c} \frac{k_{s}}{c} \sqrt{\frac{c_{f}}{2}} \\
c_{f} & =\left[2.87+1.58 \log \frac{c}{k_{s}}\right]^{-2.5}
\end{aligned}
$$

$k^{+}$is taken as a function of cascade chord length $\mathrm{C}$, chord length Reynolds number $R e_{C}$, and equivalent sand roughness $k_{s}$.

The relation between the magnitude of roughness, that is, sand grain size $k$, and equivalent sand grain roughness $k_{s}$, can be expressed as follows:

$$
k_{s}=1.5 k
$$

The boundary conditions specify a static pressure inlet $(0 \mathrm{~Pa})$, velocity outlet $(32.21 \mathrm{~m} / \mathrm{s})$, export of blade surface roughness using $k_{s}$, adiabatic and no-slip surfaces, and double-precision solution. Roughness and Reynolds number are selected as numerical simulation variables, and their parameters are shown in Table 2.

\section{MODEL VALIDATION}

\section{Cascade Wind Tunnel Experiment}

In order to ensure the accuracy of the numerical simulation results, the same cascade structure processed by three- 


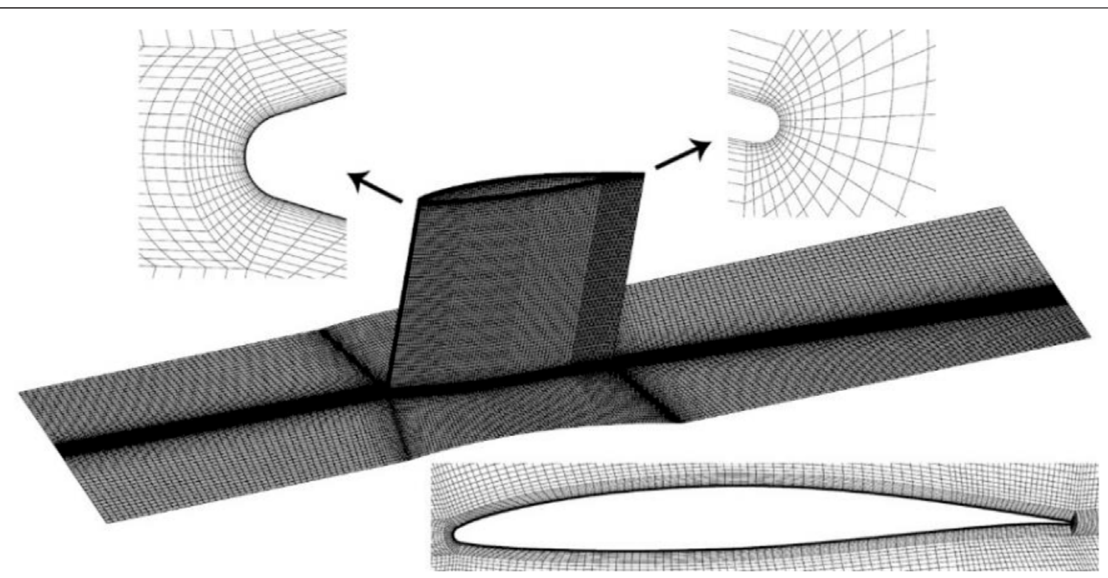

FIGURE 1 | Cascade mesh.

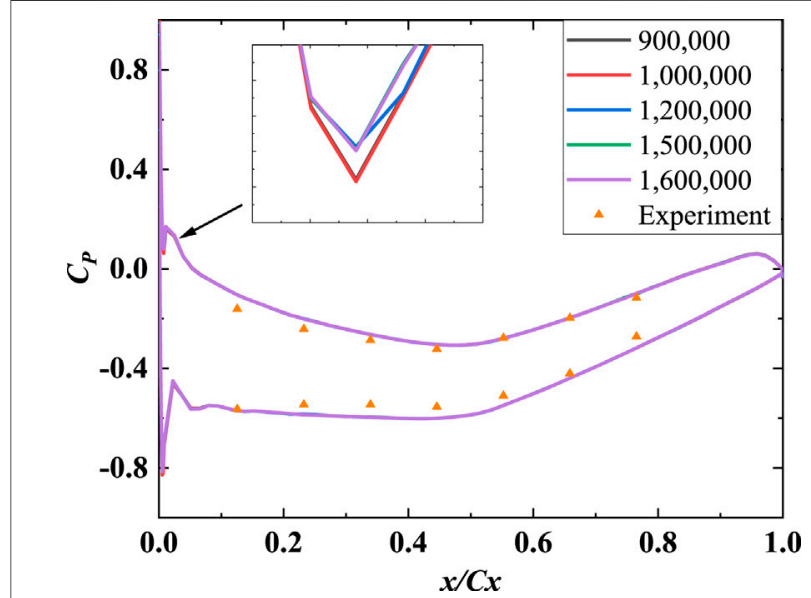

FIGURE 2 | Mesh independence validation.

TABLE 2 | Blade roughness parameters.

\begin{tabular}{lcccc}
$\mathbf{k} / \boldsymbol{\mu} \mathbf{m}$ & $\mathbf{k}_{\mathbf{s}} / \boldsymbol{\mu} \mathbf{m}$ & \multicolumn{3}{c}{$\mathbf{k}^{+}$} \\
\cline { 3 - 5 } & & $\mathbf{R e}=\mathbf{1 . 2} \times \mathbf{1 0}^{\mathbf{5}}$ & $\mathbf{R e}=\mathbf{2 . 0} \times \mathbf{1 0}^{\mathbf{5}}$ & $\mathbf{R e}=\mathbf{2 . 8} \times \mathbf{1 0}^{\mathbf{5}}$ \\
\hline 19 & 28.5 & 1.8 & 3.0 & 4.2 \\
74 & 111 & 8.1 & 13.5 & 18.9 \\
150 & 225 & 17.8 & 29.7 & 41.6 \\
180 & 270 & 21.9 & 36.5 & 51.1 \\
250 & 375 & 31.7 & 52.86 & 74.0 \\
425 & 637.5 & 57.8 & 96.4 & 135.0 \\
\hline
\end{tabular}

dimensional printing was used for experiments and compared with the simulation results. The wind tunnel test method is shown in Figure 3.

Figure 3A shows the layout diagram of the experimental. An internal variable frequency axial flow-induced draft fan provides airflow power in the experimental wind tunnel, and the different flow velocities in the wind tunnel can be achieved by adjusting the frequency of the axial flow fan. The air passes through the tapered section, the stable section, the test section, and the divergent section successively, and the above structures are all visual wind tunnel sections, made of synthetic glass. The airflow Mach number after the tapered section is less than 0.3 , so the influence of compressibility can be ignored. In order to ensure the stability of airflow, a stable section with a chord length of 3 times of the cascade is set in front of the test section.

The test section is shown in Figure 3B. Four cascades are arranged along the direction of incoming flow in the test section to ensure the same cascade consistency and reasonable blade aspect ratio. The pressure was measured on the cascade wall surface, as shown in Figure 3C. Pressure surface parameters were collected on the No.2 blade, and pressure parameters on the suction surface were collected on the No.3 blade.

\section{Cascade Roughness Treatment}

The surface roughness is changed by spraying rough particles on the cascade surface, and the sand grain size corresponds to $180 \mu \mathrm{m}$. The cascade treated with roughness is shown in Figure 4.

\section{RESULTS AND ANALYSIS Comparison of Experimental and Numerical Simulation Results}

The experiment and numerical simulations under the same conditions were carried out under the smooth wall surface and a $180 \mu \mathrm{m}$ roughness cascade. The comparison results are shown in Figure 5. When the cascade is a smooth wall, it can be observed that the numerical simulation results agree well with the experimental prediction, and the maximum error is less than $5 \%$. When the cascade surface is rough, the experimental data showed that the static pressure coefficient of the cascade surface decreased, and numerical simulation of the same $\gamma-R_{\theta}$ transition model also captures the static pressure coefficient. Overall, the numerical results correspond well with the test results, which can meet the prediction and mechanism 
A Current collector

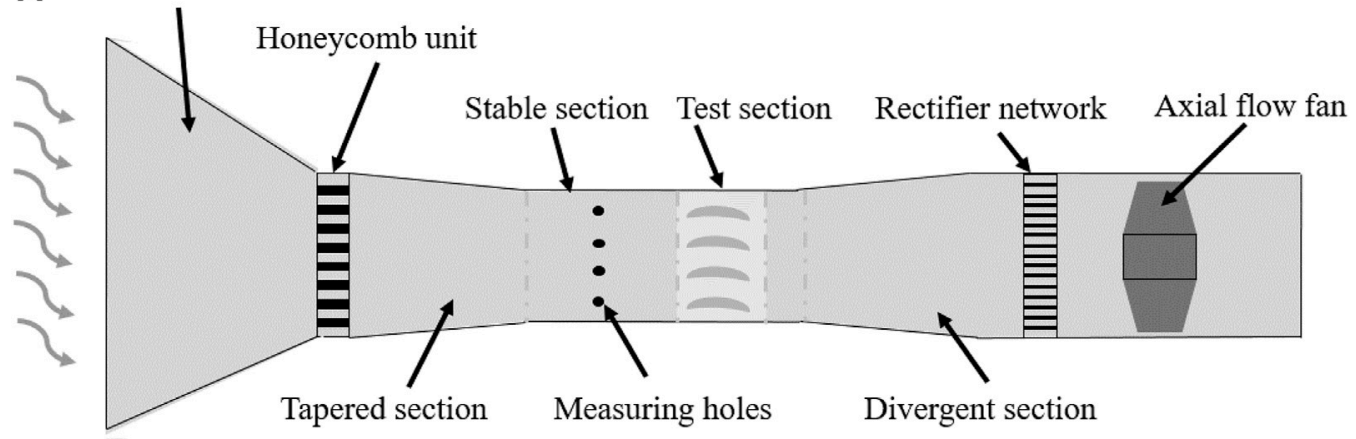

B

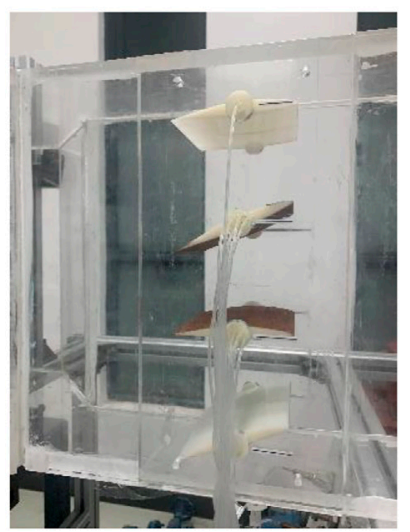

C

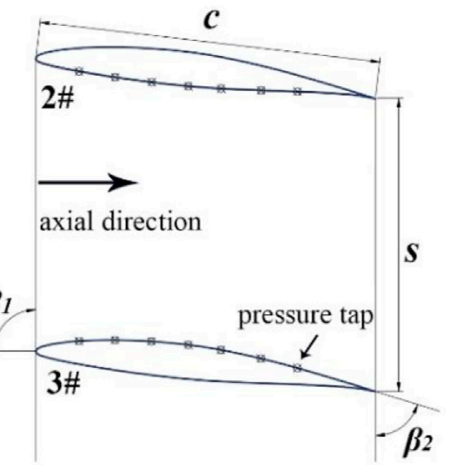

FIGURE 3 | Experimental test pattern: (A) test schematic diagram, (B) test section, and (C) cascade structure and pressure measuring points.

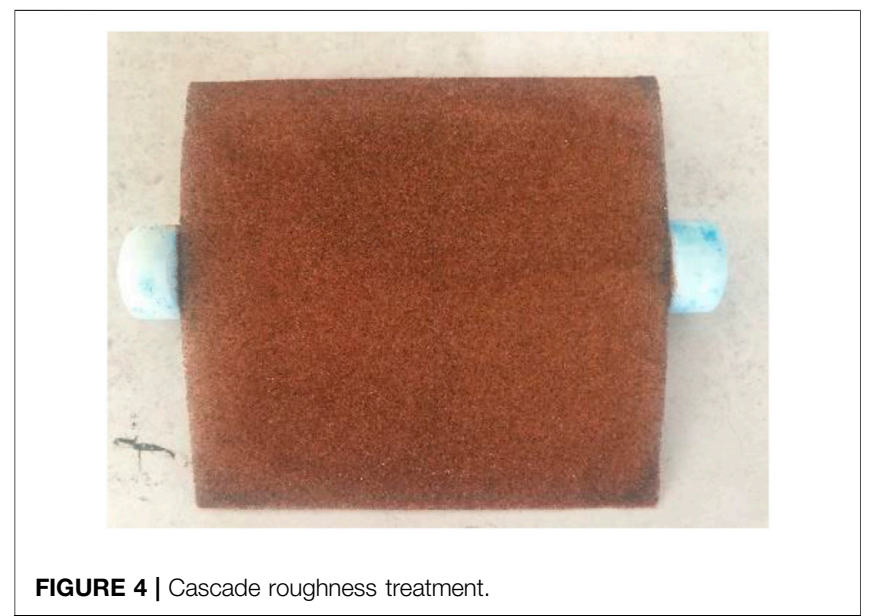

analysis of performance loss and flow characteristics under the change of cascade roughness.

\section{Boundary Layer Transition}

Roughness can influence the flow characteristics in the boundary layer to a certain extent, and the effect of roughness on the flow in the boundary layer also changes with the change of Reynolds

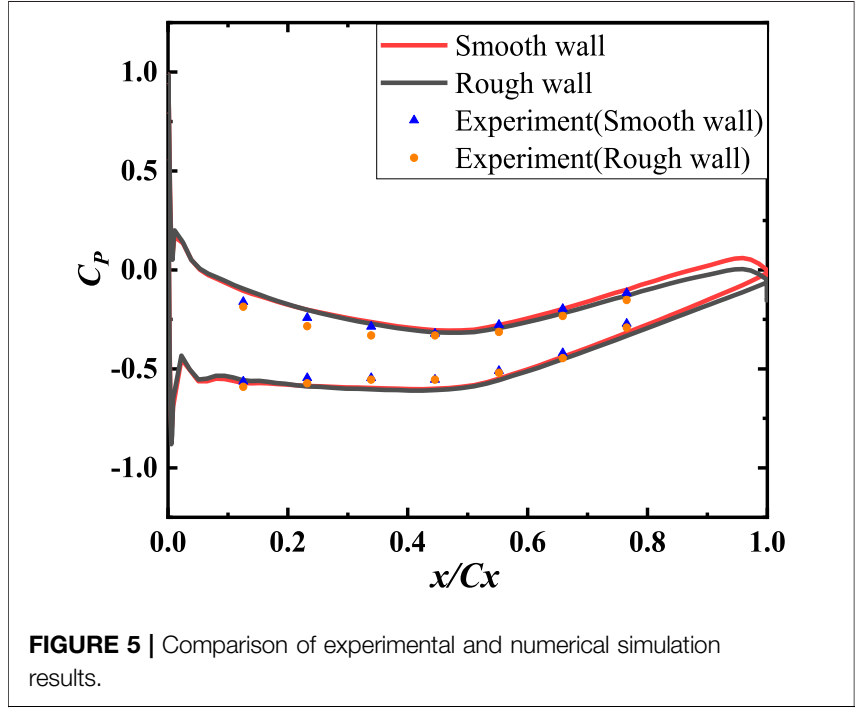

number. Boundary layer development can be analyzed by the friction resistance coefficient of the cascade surface transition. The friction resistance coefficient is an important parameter in boundary laminar flow, which can be used to characterize the 


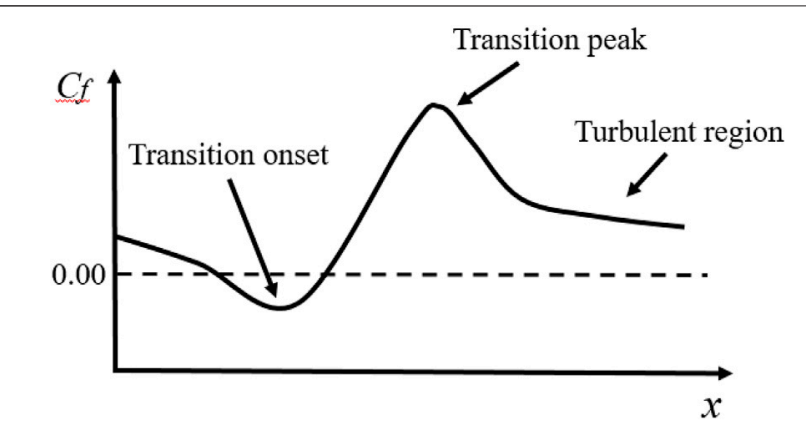

FIGURE 6 | Schematic diagram of the transition process.

viscous shear stress on the wall and the transition process in the boundary layer flow, and the change of roughness is closely related to the viscous loss on the wall. The variation process of turbulence in the boundary layer can also be used as evidence to predict boundary layer transition.

As shown in Figure 6 (Schlichting, 1979; Bammert and Milsch, 1972), the dotted line in the friction coefficient distribution diagram on the suction surface indicates that the friction coefficient $\left(C_{f}\right)$ is 0 ; if the friction coefficient is less than 0 , it indicates that the suction surface of the cascade appears as a countercurrent area, and it presents flow separation. The separation point of the flow and the flow re-attachment point at the end of the separation bubble can be determined, and the length of the separation bubble can be obtained, that is, the distance between the two intersection points with $C_{f}=0$.

Figure 7 shows the distribution of friction coefficient and turbulence intensity on the suction surface of the cascade with different roughnesses when the Reynolds number is $2.0 \times 10^{5}$.

When the cascade is smooth, separation bubbles appear from the cascade leading edge to $0.07 \mathrm{Cx}$. The fluid is disturbed by the increase of roughness, and the turbulence intensity increases rapidly from the leading edge. The fluid gains the energy to resist separation, which reduces the length of the separation bubble to a certain extent, but the roughness height is too small to completely inhibit separation.
When the surface roughness is $74 \mu \mathrm{m}$, the separation bubbles are inhibited until they almost disappear. When the surface roughness is increased to $150 \mu \mathrm{m}$, the separation bubbles disappear. It shows that the increase of roughness has a certain inhibitory effect on the formation of separation bubbles. When the roughness is between 74 and $150 \mu \mathrm{m}$, the disturbance of the roughness to the boundary layer makes the separation bubble disappear and the separation loss caused by the separation bubble decreases.

In general, in the process of roughness increase, the growth positions and peak values of friction resistance coefficient and turbulence intensity change little. It indicates that the transition starting position does not change much, but the disturbance to the flow increases, the turbulence intensity in the leading-edge area increases, and the growth rate of turbulence intensity before reaching the peak is also higher, which makes the flow more resistant to separation.

\section{Boundary Layer Development at Different Reynolds Numbers}

As can be seen from Figure 8, the variation of Reynolds number on smooth cascades has little influence on friction coefficient of the suction surface. When the roughness increases to $74 \mu \mathrm{m}$, the friction coefficient at the cascade leading edge increases in advance with a higher Reynolds number, and the separation bubbles disappear at the cascade leading edge with a Reynolds number of $2.8 \times 10^{5}$. When the roughness is $180 \mu \mathrm{m}$, it shows that the larger the Reynolds number is, the larger the friction coefficient is, and the earlier the position appears.

The increasing rate of turbulence increases with the increase of roughness. Under the same roughness, when the Reynolds number is larger, the transition position is advanced and the transition process is faster. The influence of Reynolds number increases with the increase of roughness.

Figure 9 shows the distribution clouds of friction coefficient and turbulence on the suction surface of cascades with different Reynolds numbers $\left(1.2 \times 10^{5}\right.$ and $\left.2.8 \times 10^{5}\right)$ and a surface roughness of $74 \mu \mathrm{m}$. Under the conditions of low Reynolds numbers, the area of low friction drag coefficient and turbulence intensity between the cascade
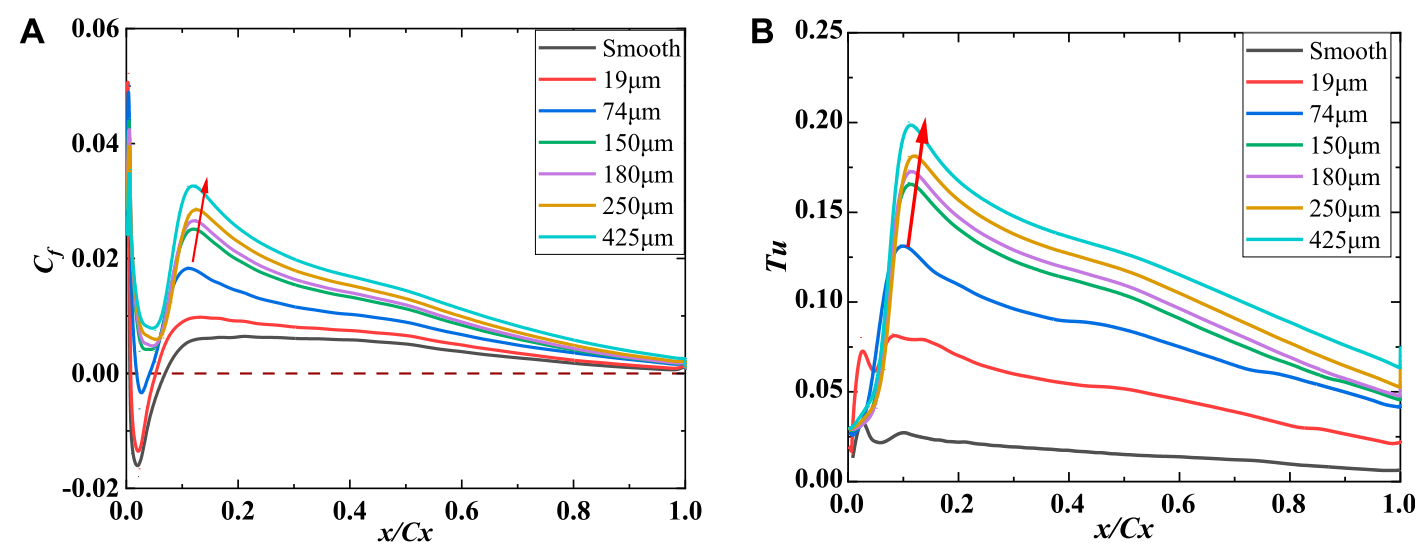

FIGURE 7| Axial friction coefficient and turbulence level distribution of different roughnesses: (A) coefficient of axial friction resistance and (B) turbulence intensity. 


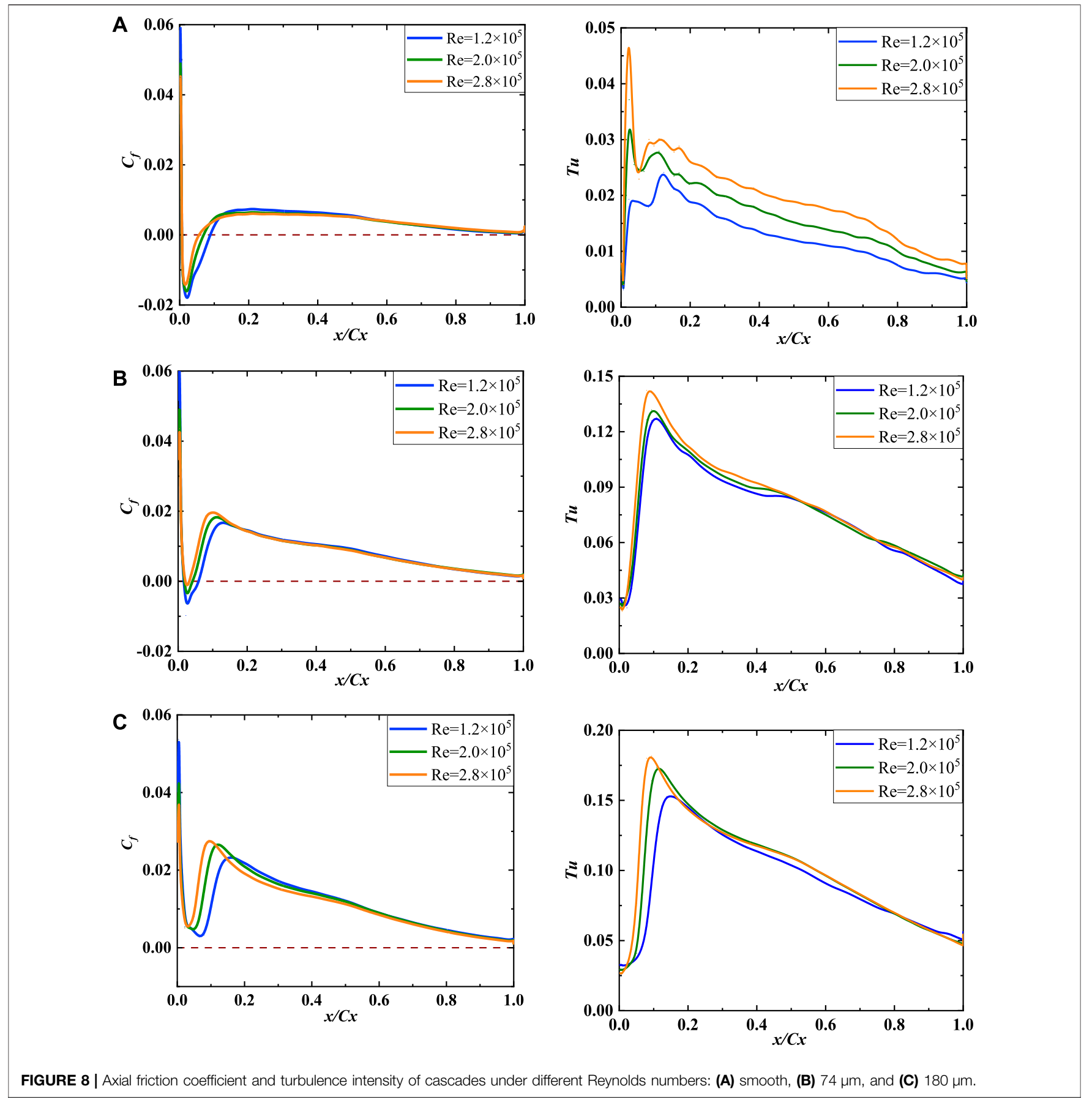

leading edge and the trailing edge corner is larger than that of the high Reynolds number. The reason is that the separation bubble size of the cascade leading edge is larger under the conditions of low Reynolds numbers, and the low energy fluid accumulation degree in the trailing edge corner area is larger, and the separation range in the corner area is larger. When the Reynolds number is larger, the position of transition moves forward, and the the transition process is faster. However, the change of Reynolds number has little influence on the transition characteristics and forms of the suction surface.

\section{Effects of Cascade Losses at Different Reynolds Numbers}

Figure 7 shows that there is a closed leading edge separation bubble on the suction surface of the cascade. As the roughness 

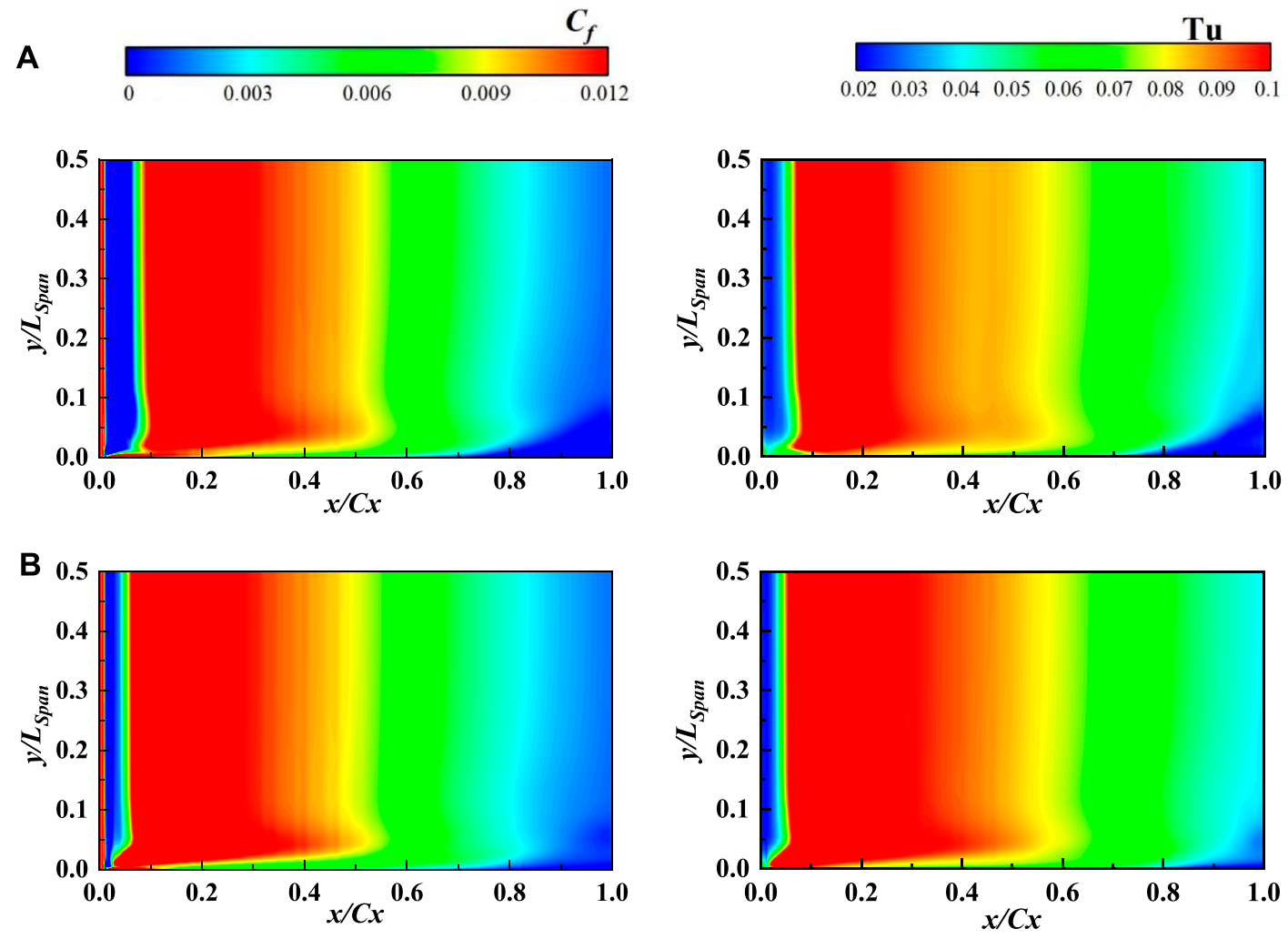

FIGURE 9 |Distribution cloud diagram of frictional resistance coefficient and turbulence intensity on the suction surface at different Reynolds numbers: (A) Re = 1.2 $\times 10^{5}$ and $(\mathbf{B}) \operatorname{Re}=2.8 \times 10^{5}$.

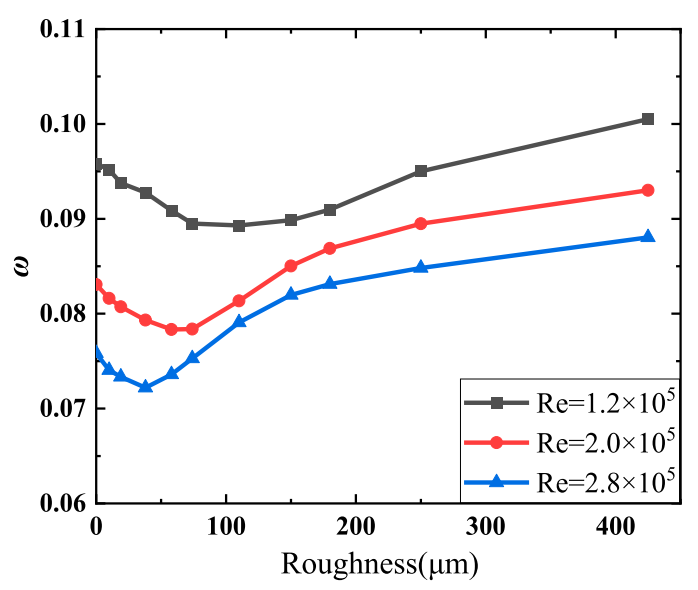

FIGURE 10 | Effect of roughness on cascade total pressure loss at different Reynolds numbers.

increases, the roughness inhibits the leading-edge separation bubble and reduces the separation loss. However, as the roughness increases, the wall viscosity loss continues to increase. In this article, the effects of roughness on cascade losses under different Reynolds numbers $\left(1.2 \times 10^{5}, 2.0 \times 10^{5}\right.$,
$2.8 \times 10^{5}$ ) are analyzed in detail, and the roughness-sensitive areas of cascades under different roughness can be accurately observed.

Researchers (Bammert and Milsch, 1972; Syverud and Bakken, 2006) usually use the total pressure loss coefficient to represent the cascade loss, which is defined as follows:

$$
\omega=\frac{P_{T, \text { in }}-P_{T, \text { out }}}{P_{T, \text { in }}-P_{\text {in }}}
$$

where $P_{T, \text { in }}$ and $P_{\text {in }}$ represent the total pressure at the inlet and the static pressure at the inlet, respectively, and $P_{T, \text { out }}$ represents the total pressure at the outlet.

Figure 10 shows the effect of roughness on the cascade total pressure loss at different Reynolds numbers. Under different Reynolds numbers, the total pressure loss decreases first and then increases with the roughness; however, the sensitive area of loss changes with different Reynolds numbers. In the process of roughness increase, the variation ranges of cascade total pressure loss increase with the increase of the Reynolds number. In sensitive areas, roughness has a greater influence on cascade loss.

\section{Variation of Static Pressure Coefficient at Different Reynolds Numbers}

Figure 11 shows the static pressure coefficient distribution on the cascade surface. According to the static pressure coefficient changes under different roughnesses, the effect of Reynolds 

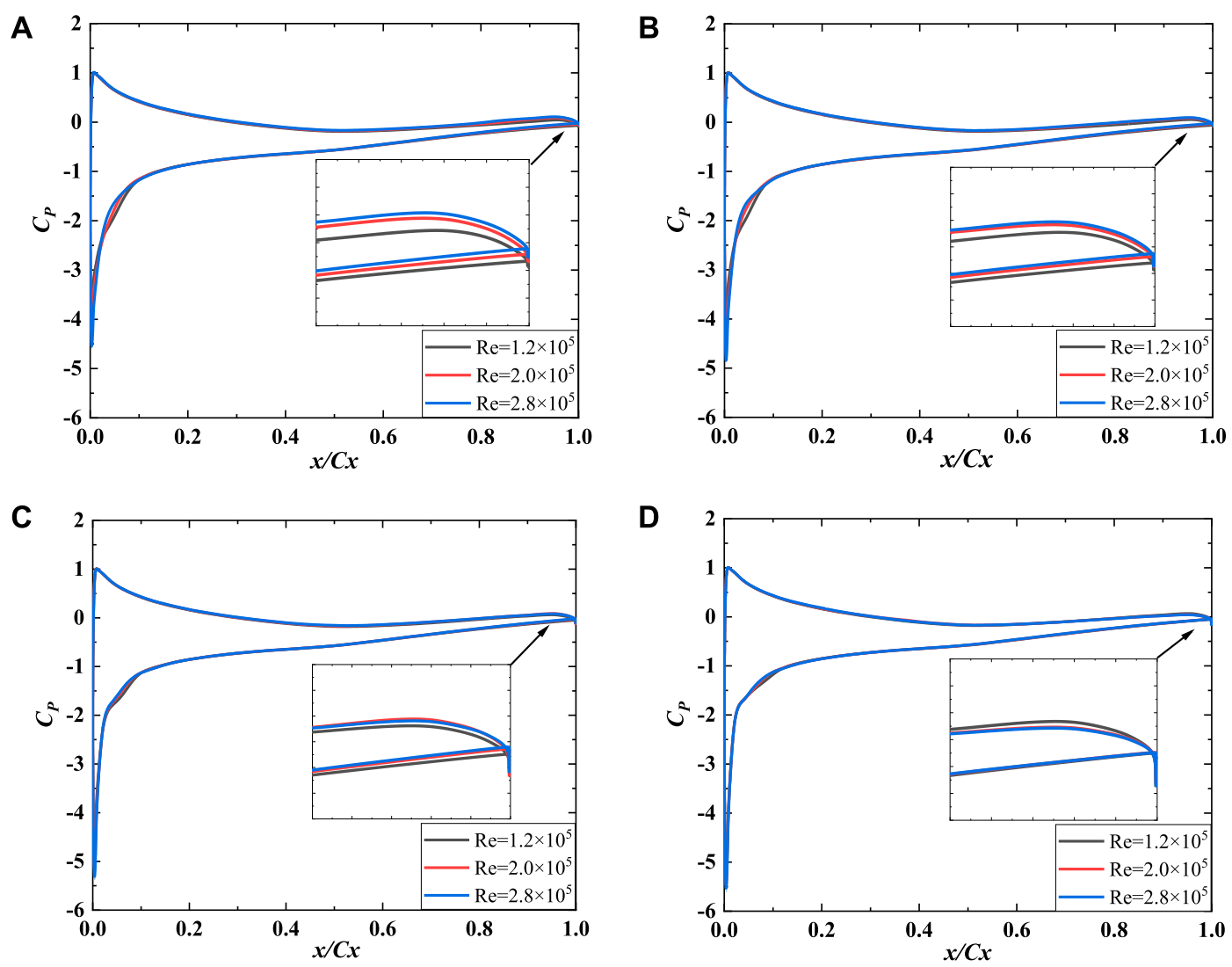

FIGURE 11 | Effect of roughness on surface static pressure distribution at different Reynolds numbers: (A) smooth, (B) $19 \mu \mathrm{m}$, (C) $74 \mu \mathrm{m}$, and (D) $180 \mu \mathrm{m}$.

number on the cascade is mainly manifested in two parts. The first part is located at the leading edge of the suction surface of the cascade, where there are separation bubbles, indicating that the higher the Reynolds number is, the higher the static pressure coefficient is. The other part is located in the rear section of the cascade. The larger the Reynolds number is, the larger the static pressure coefficient of the rear section of the cascade is. With the increase of roughness, the difference between the front edge and the back section of cascade suction gradually decreases, which also confirms the inhibition effect of roughness increase on the suction front edge separation bubble in Figure 7.

\section{Effects of Wake Loss}

Figure 12 shows the influence of cascade wake loss. Considering the wake loss under different roughnesses, the effect of Reynolds number on wake loss varies. The wake loss of smooth cascades decreases with the increase of Reynolds number, and the width of wake loss decreases accordingly. The effect of Reynolds number changes with the increase of roughness. When the roughness increases to $180 \mu \mathrm{m}$, the cascade wake loss increases gradually with the increase of Reynolds number, and the wake loss is close to the pressure side. When the Reynolds number is $1.2 \times 10^{5}$, the wake loss decreases with the increase of roughness, and the decrease in the amplitude increases. When the Reynolds number is $2.0 \times 10^{5}$ and $2.8 \times 10^{5}$, the wake loss decreases first and then increases with increasing roughness. This is mainly because the cascade boundary layer is relatively thin, and flow separation has been inhibited at a small roughness. The wake loss will continue to increase with increasing roughness.

\section{Influence of Outlet Flow Angle}

Figure 13 shows the spanwise change curve of the outlet flow angle at $0.2 C x$ from the cascade. Under different roughnesses, there are different variation trends. The outlet flow angle of smooth cascades varies significantly with different Reynolds numbers, but the flow angle distribution in the high part of the middle cascade is gradually stable. With the increase of Reynolds number, the length of middle blade high stability area decreases gradually. With the increase of Reynolds number, the influence range of flow in the area near the end wall of the smooth cascade increases, and the instability range of flow angle behind the cascade near the end wall increases.

When the Reynolds number is $1.2 \times 10^{5}$, the flow outlet angle of the smooth cascade varies uniformly along with the cascade height from about 0.2 Span to 0.8 Span. When the surface roughness increases to $19 \mu \mathrm{m}$, the difference between the middle blade height region and the flow angle near the end wall becomes larger. When the surface roughness increases to $74 \mu \mathrm{m}$, it changes dramatically between the cascade end wall and 0.2 Span. When the roughness is increased to $180 \mu \mathrm{m}$, the flow 

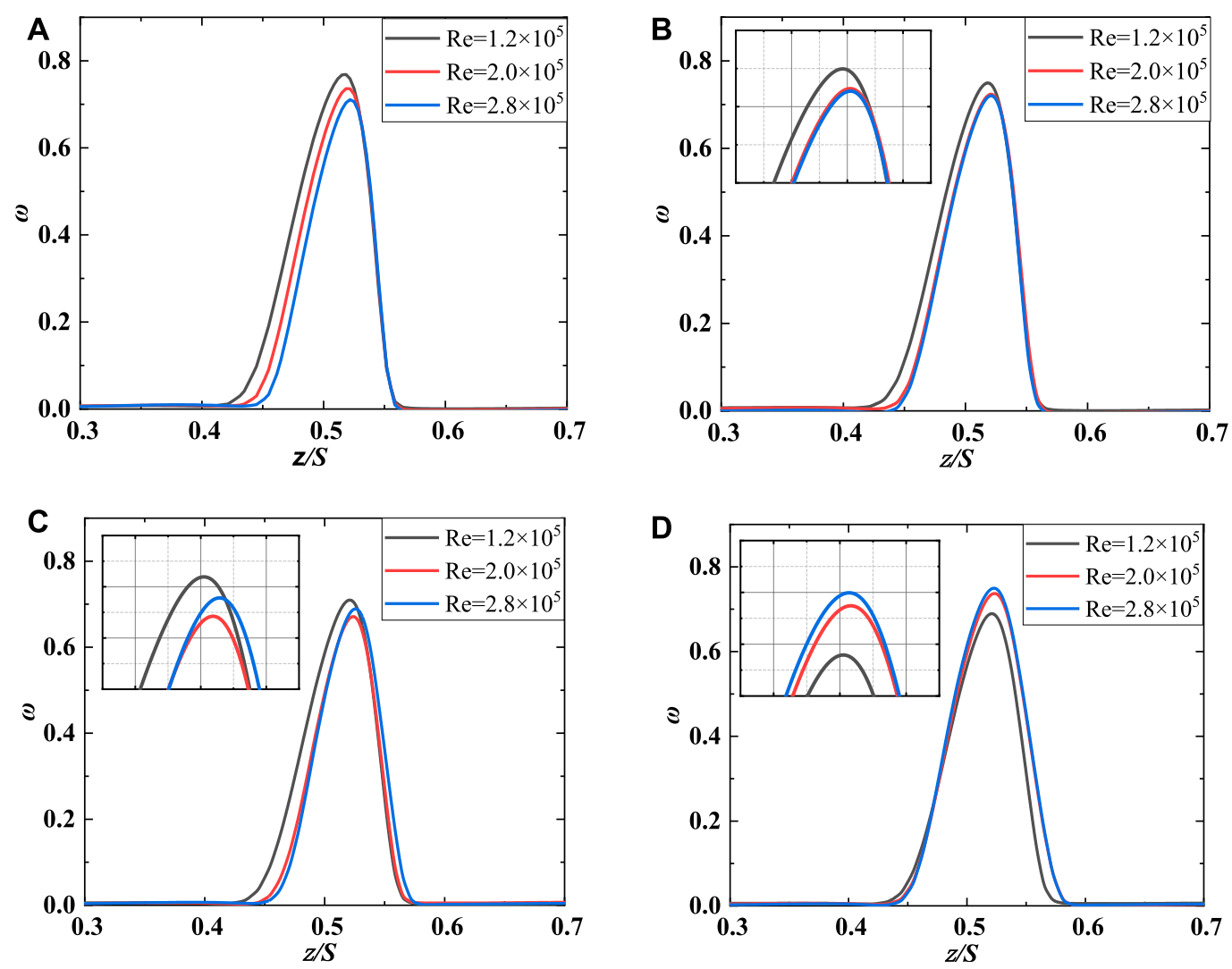

FIGURE 12 | Effects of roughness on cascade wake loss at different Reynolds numbers: (A) smooth, (B) $19 \mu \mathrm{m}$, (C) $74 \mu \mathrm{m}$, and (D) $180 \mu \mathrm{m}$.

angle curves near the end wall of the cascade are close to coincidence. With the decrease of Reynolds number, the flow angle of the middle blade height at the cascade position decreases gradually, and the difference of the flow angle between the end wall region of the cascade and the middle blade height region of the cascade increases.

Overall, the increase of roughness increases the aerodynamic loss of the cascade and decreases the efficiency of the cascade. However, when flow separation occurs on the suction surface of the cascade, roughness affects the boundary layer flow and thus inhibits flow separation, thus reducing the cascade separation loss to a certain extent. The viscosity loss increases with the increase of roughness, while the separation loss decreases within a certain range of surface roughness.

\section{Effect of the Suction Boundary Layer on the Cascade at Different Reynolds Numbers}

In order to further describe the influence of roughness on the boundary layer on the suction surface, it is necessary to analyze the thickness of the boundary layer and some parameters in the boundary layer, such as displacement thickness, momentum thickness, shape factor, etc.

The displacement thickness $\delta^{*}$ in the actual fluid is blocked due to its own viscosity, resulting in the decrease of the flow at this position, which is equivalent to the distance from the wall to the normal outward movement of the outflow in the process of the ideal fluid flowing around the wall; that is, the flow shape around the wall is equal to the wall shape plus the displacement thickness of the boundary layer

$$
\delta^{*}=\int_{0}^{\delta^{*}}\left(1-\frac{u\left(y_{n}\right)}{U_{x}}\right) d y_{n}
$$

where $y_{n}$ represents the local height along the wall normal, $u\left(y_{n}\right)$ represents the actual flow velocity at $y_{n}$, and $U_{x}$ represents the local free flow velocity. The integrals are all within the actual boundary layer thickness.

The momentum thickness, also known as loss thickness, is the momentum lost in the boundary layer due to the viscosity of the fluid itself. The flow state of the boundary layer is usually judged 

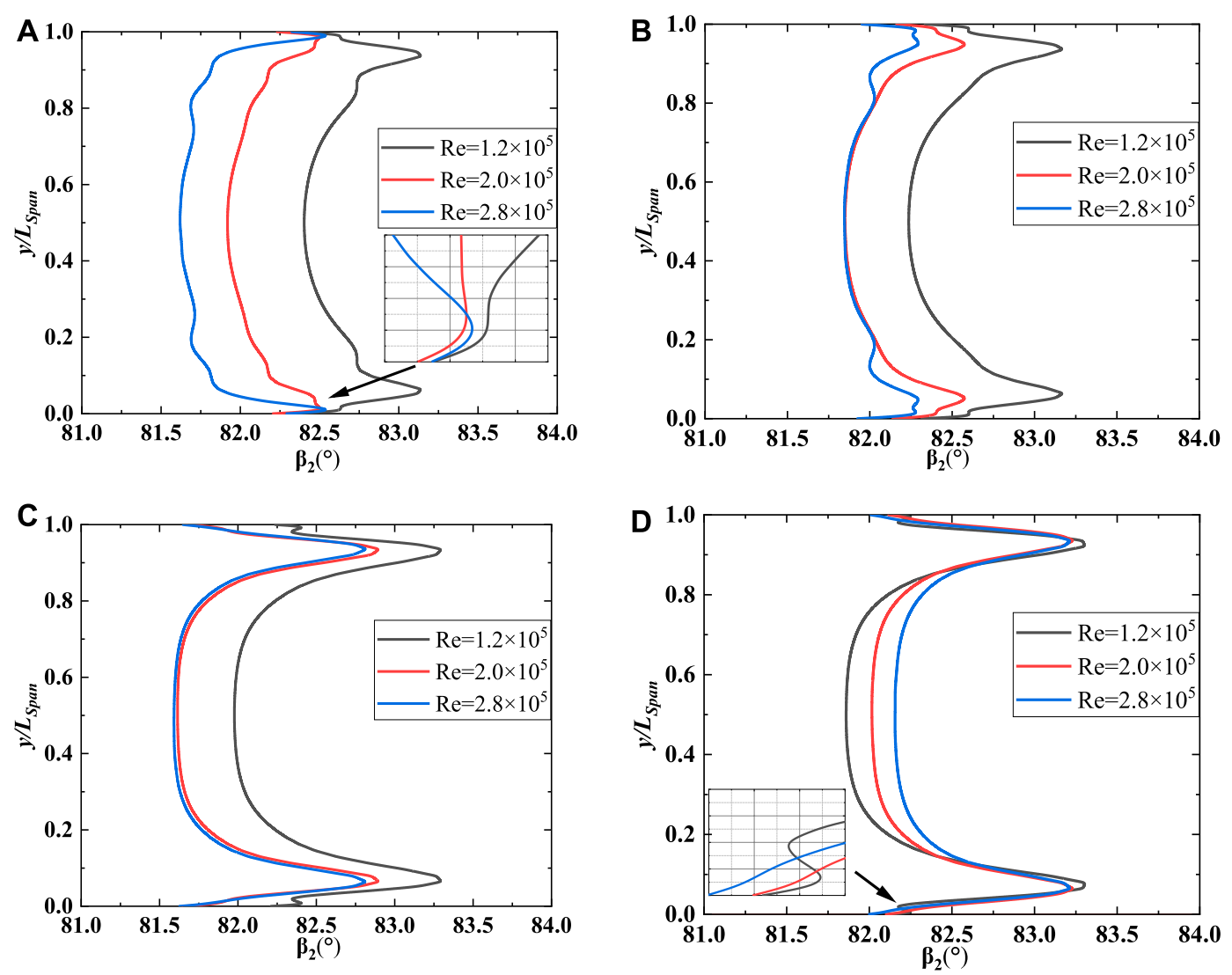

FIGURE 13 | Effect of roughness on outlet flow angle at different Reynolds numbers: (A) smooth, (B) $19 \mu \mathrm{m}$, (C) $74 \mu \mathrm{m}$, and (D) $180 \mu \mathrm{m}$.

by observing the shape change of the velocity profile of the boundary layer

$$
\theta^{*}=\int_{0}^{\delta^{*}} \frac{u\left(y_{n}\right)}{U_{x}}\left(1-\frac{u\left(y_{n}\right)}{U_{x}}\right) d y_{n}
$$

It is difficult to quantitatively compare the boundary layer velocity profiles under different working conditions directly with the momentum thickness. In order to quantitatively describe the boundary layer shape, the shape factor $H_{12}$ is often used to describe the boundary layer velocity profile. Because its value is the ratio of displacement thickness to momentum thickness, the smaller the shape factor is, the fuller the boundary layer velocity profile is. When $H_{12}=1$, it indicates that the boundary layer velocity is the fullest. The shape factors of the turbulent boundary layer and laminar boundary layer will change greatly

$$
H_{12}=\frac{\delta^{*}}{\theta^{*}}
$$

Figure 14A shows the distribution of boundary layer thickness on the suction surface of the cascade with different roughnesses under $\operatorname{Re}=2.0 \times 10^{5}$. Through the change of boundary layer thickness, it can be observed that the boundary layer at the front edge of the cascade suction appears as an obvious hump, and the bubbles at the front edge disappear after increasing the roughness. The $19 \mu \mathrm{m}$ roughness has a great effect on the cascade boundary layer and inhibits the bubbles in the leading edge. In general, the thickness of the leading-edge boundary layer decreases with the increase of roughness. With the increase of roughness, the boundary layer thickness shows a trend of decreasing first and then increasing at $0.45 \mathrm{Cx}$, but the overall boundary layer thickness has little change.

Figure 14B shows the displacement thicknesses of cascades with different roughnesses. The distribution of $\delta^{*}$ can accurately show the position of separation bubbles and reflect the displacement effect produced by separation bubbles at the leading edge. With the increase of roughness, the separation bubble is restrained, the displacement effect is weakened, and the growth rate of the boundary layer decreases. Figure 14C shows the change of momentum thickness, which refers to the momentum loss in the boundary layer. The separation bubbles appear in the smooth and small roughness cascade leading edge, resulting in a large loss.

The boundary layer parameter displacement thickness and momentum thickness show a decreasing trend at the leading edge with increasing roughness. With the increase of roughness, the displacement thickness and momentum thickness in the second 

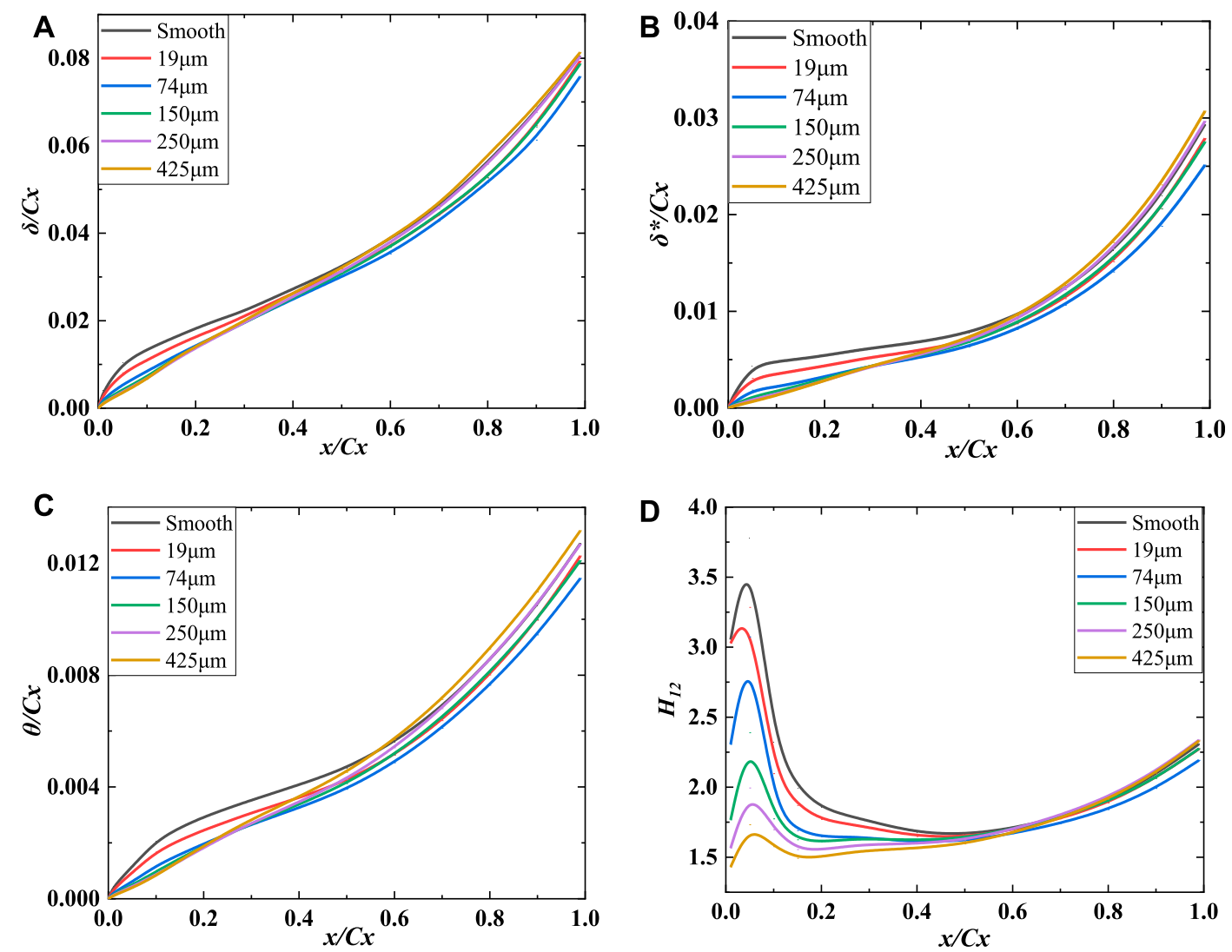

FIGURE 14 | Influence of roughness on the boundary layer parameters of the suction surface: (A) boundary layer thickness, (B) displacement thickness, (C) momentum thickness, and (D) shape factor.

half of the cascade decrease first and then increase. When the roughness is $74 \mu \mathrm{m}$, the displacement thickness and momentum thickness at the second half of the cascade are the minimum.

The shape factor in Figure 14D can not only judge the distribution of the boundary layer velocity shape but also be used to judge laminar flow and turbulent flow. The shape factor at the leading edge of the cascade decreases with the increase of roughness, which means that the velocity profile at this position becomes fuller. At the leading edge of the cascade, there is a protrusion of shape factor at $0.05 \mathrm{Cx}$, which develops slightly backward with the increase of roughness. The shape factors of different roughnesses after front bumps all have varying degrees of decline, in a smooth or roughness is small, frontal area separation bubble still exists, the shape factor is higher, and drops rapidly after protrusions, performance from the layer of flow turbulence transition process, with fluid flow, the cascade after a period of rising. Combined with the change of wake loss in the previous section, when the roughness is $74 \mu \mathrm{m}$, the shape factor of the rear section of the cascade is the smallest, and it indicated that the appropriate roughness can not only suppress the leading-edge separation bubble but also optimize the boundary layer flow pattern and reduce the flow blocking and mixing loss at the trailing edge of the cascade.

\section{CONCLUSION}

In this article, based on the research object of NACA-65 cascades, the reliability of numerical simulation is verified through experiments at the first. Second, the influence of roughness size on cascade boundary layer flow under different Reynolds numbers is studied by the numerical simulation method. The main conclusions are as follows:

1) There are separation bubbles at the suction front edge of smooth cascades, and the increase of roughness can inhibit the separation bubbles. With the increase of roughness, the turbulence intensity in the leading edge region increases, the ability of airflow to resist separation is stronger, the transition process is accelerated, the separation bubble gradually disappears, and the separation loss is reduced.

2) With the increase of roughness, the total cascade loss first decreases and then increases. With the increase of roughness, the total cascade loss appears in the sensitive region, and roughness has a greater effect on cascade loss in the sensitive region. With the increase of roughness, the effect of static pressure coefficient on the cascade surface increases, and the main difference is reflected in the position of the front edge and back section of cascade suction. 
3) At a low Reynolds number, the cascade wake loss increases gradually. With the increase of Reynolds number, the wake loss first decreases and then increases, and the range of wake loss is closer to the pressure side.

4) With the increase of roughness, the boundary layer thickness shows a trend of decreasing first and then increasing from $0.45 C x$, but the overall boundary layer thickness has little change. The displacement thickness and momentum thickness at the leading edge of the cascade decrease with the increase of roughness and show a trend of decreasing first and then increasing in the latter half. When the roughness is $74 \mu \mathrm{m}$, the displacement thickness, momentum thickness, and shape factor at the back of the cascade are the minimum (Wu and Piomelli, 2018; Rodriguez et al., 2017; Butler and Wu, 2018; Nyantekyi-Kwakye et al., 2019; Mendonca and Sharif, 2010; Xu et al., 2020; Zhou et al., 2021; Spalart and Watmuff, 1993; Li et al., 2020; Lee et al., 2018; Schlichting, 1936; Atmani et al., 2009; Goldfeld and Orlik, 2005; Back et al., 2009).

\section{REFERENCES}

Atmani, R., Brima, A., and Askovic, R. (2009). Study of Three-Dimensional Separation of Boundary Layer over Blunt Bodies. J. Hydrodynamics 21 (01), 100-107. CNKI:SUN:SDYW.0.2009-01-016. doi:10.1016/s1001-6058(08) 60124-9

Back, S. C., Jeong, I. C., Sohn, J. L., and Song, S. J. (2009). Influence of Surface Roughness on the Performance of a Compressor Blade in a Linear Cascade: Experiment and ModelingASME Turbo Expo 2009: Power for Land, Sea, and Air. American Society of Mechanical Engineers.

Back, S. C., Hobson, G. V., Millsaps, S. J., and Millsaps Knox, T. (2012). Effects of Reynolds Number and Surface Roughness Magnitude and Location on Compressor Cascade Performance. J. Turbomach. 134 (5), 051013. doi: $10.1115 / 1.4003821$

Bammert, K., and Milsch, R. (1972). Boundary Layers on Rough Compressor bladesMechanical Engineering. American Society of Mechanical Engineers.

Boese, M., and Fottner, L. (2002). Effects of Riblets on the Loss Behavior of a Highly Loaded Compressor Cascade. Amsterdam: American Society of Mechanical Engineers.

Butler, A., and Wu, X. (2018). Stationary Crossflow Vortices Near the Leading Edge of Three-Dimensional Boundary Layers: the Role of Non-parallelism and Excitation by Surface Roughness. J. Fluid Mech. 845, 93-140. doi:10.1017/ jfm.2018.226

Gbadebo, S. A., Hynes, T. P., and Cumpsty, N. A. (2004). Influence of Surface Roughness on Three-Dimensional Separation in Axial Compressors. J. Turbomach. 126, 455-463. doi:10.1115/1.1791281

Goldfeld, M. A., and Orlik, E. V. (2005). The Research of Laminar-Turbulent Transition in Hypersonic Three-Dimensional Boundary Layer. J. Therm. Sci. 14 (02), 103-107. CNKI:SUN:RKXY.0.2005-02-002. doi:10.1007/s11630-0050018-y

Ishida, M., Ueki, D., and Hironobu, U. (2001). Suppression of Rotating Stall by Wall Roughness Control in Vaneless Diffusers of Centrifugal Blowers. J. Turbomach. 123, 64-72. doi:10.1115/1.1328084

Khalfallah, M. G., and Koliub, A. M. (2007). Effect of Dust on the Performance of Wind Turbines. Desalination 209, 209-220. doi:10.1016/j.desal.2007.04.030

Koch, C. C., and Smith, L. H. (1980). Discussion: "Experimental and Analytical Investigation of the Effects of Reynolds Number and Blade Surface Roughness on Multistage Axial Flow Compressors" (Scha"ffler, A., 1980, ASME J. Eng. Power, 102, Pp. 5-12). J. Eng. Power 102, 12. doi:10.1115/1.3230208

Lee, J., Kim, H., and Park, H. (2018). Effects of Superhydrophobic Surfaces on the Flow Around an NACA0012 Hydrofoil at Low Reynolds Numbers. Exp. Fluids 59 (7), 111. doi:10.1007/s00348-018-2564-6

\section{DATA AVAILABILITY STATEMENT}

The original contributions presented in the study are included in the article/supplementary material; further inquiries can be directed to the corresponding authors.

\section{AUTHOR CONTRIBUTIONS}

YL and ZZ provided experimental ideas and theoretical guidance. PW provided language guidance and writing guidance. All authors contributed to the article and approved the submitted version.

\section{FUNDING}

This work was supported by the National Natural Science Foundation of China (Grant No. 51776217).

Leipold, R., Fottner, M., and Leonhard, F. (2000). The Influence of Technical Surface Roughness Caused by Precision Forging on the Flow Around a Highly Loaded Compressor Cascade. J. Turbomach. 122, 416-424. doi:10.1115/ 1.1302286

Li, Q. a., Kamada, Y., Maeda, T., and Yamada, K. (2020). Investigations of Flow Field Around Two-Dimensional Simplified Models with Wind Tunnel Experiments. Renew. Energ. 152, 270-282. doi:10.1016/ j.renene.2019.12.046

Mendonca, J., and Sharif, M. A. R. (2010). Turbulent Transonic Flow over Smooth and Rough Circular Arc Bumps in a Freestream. Eng. Appl. Comput. Fluid Mech. 4 (2), 287-300. doi:10.1080/19942060.2010.11015317

Nikuradse, J. (1950). Laws of Flow in Rough Pipes. Washington: National Advisory Committee forAeronautics.

Nyantekyi-Kwakye, B., Pahlavan, H., Clark, S. P., Tachie, M. F., and Dow, K. (2019). Roughness Effect on Turbulent Flow Structure beneath a Simulated Ice Jam. J. Hydraulic Res. 57 (2), 238-249. doi:10.1080/ 00221686.2018.1473298

Rodriguez, I., Lehmkuhl, O., Piomelli, U., Chiva, J., Borrell, R., and Oliva, A. (2017). LES-based Study of the Roughness Effects on the Wake of a Circular Cylinder from Subcritical to Transcritical Reynolds Numbers. Flow Turbulence Combust 99, 729-763. doi:10.1007/s10494-017-9866-2

Scha"ffler, A. (1980). Experimental and Analytical Investigation of the Effects of Reynolds Number and Blade Surface Roughness on Multistage Axial Flow Compressors. J. Eng. Power 102, 5-12. doi:10.1115/1.3230232

Schlichting, H. (1936). Experimental Investigation of the Problem of Surface Roughness. NACA-TM-823.

Schlichting, H. (1979). Boundary-Layer Theory 7. McGraw-Hill.

Schreiber, H.-A., Steinert, W., and Ku"sters, B. (2002). Effects of Reynolds Number and Free-Stream Turbulence on Boundary Layer Transition in a Compressor Cascade. J. Turbomach. 124, 1-9. doi:10.1115/1.1413471

Shamsoddin, S., and Porté-Agel, F. (2017). Large-Eddy Simulation of Atmospheric Boundary-Layer Flow through a Wind Farm Sited on Topography. Boundary-Layer Meteorology 163, 1-17. doi:10.1007/ s10546-016-0216-z

Spalart, P. R., and Watmuff, J. H. (1993). Experimental and Numerical Study of a Turbulent Boundary Layer with Pressure Gradients. J. FZuidMeeh 249, 337-371. doi:10.1017/s002211209300120x

Syverud, E., and Bakken, L. E. (2006). The Impact of Surface Roughness on Axial Compressorperformance deteriorationASME Turbo Expo 2006: Power for Land, Sea, and Air. American Society of Mechanical Engineers.

Tani, I., and Hama, F. R. (1953). Some Experiments on the Effect of a Single Roughness Element on Boundary-Layer Transition. J. Aeronaut. Sci. 20 (4), 289-290. doi:10.2514/8.2613 
Tarabrin, A. P., Schurovsky, V. A., Bodrov, A. I., and Stalder, J.-P. (1998). An Analysis of Axial Compressor Fouling and a Blade Cleaning Method. J. Turbomach. 120 (2), 256-261. doi:10.1115/1.2841400

Tarabrin, A. P., Schurovsky, V. A., Bodrov, A. I., and Stalder, J. P. (1998). Influence of Axial Compressor Fouling on Gas Turbine Unit Performance Based on Different Schemes and with Different Initial Parameters. Stockholm, Sweden: International Gas Turbine \& Aeroengine Congress \& Exhibition.

Wu, W., and Piomelli, U. (2018). Effects of Surface Roughness on a Separating Turbulent Boundary Layer. J. Fluid Mech. 841, 552-580. doi:10.1017/ jfm.2018.101

Xu, F., Zhong, S., and Zhang, S. (2020). Experimental Study on Secondary Flow in Turbulent Boundary Layer over Spanwise Heterogeneous Microgrooves. Phys. Fluids 32, 035109. doi:10.1063/1.5142727

Zhou, H., Zhu, Y., Tian, G., Feng, X., and Zhang, Y. (2021). Experimental Investigations of the Turbulent Boundary Layer for Biomimetic Surface with Spine-Covered Protrusion Inspired by Pufferfish Skin. Arab J. Sci. Eng. 46, 2865-2875. doi:10.1007/S13369-020-05235-6
Conflict of Interest: The authors declare that the research was conducted in the absence of any commercial or financial relationships that could be construed as a potential conflict of interest.

Publisher's Note: All claims expressed in this article are solely those of the authors and do not necessarily represent those of their affiliated organizations or those of the publisher, the editors, and the reviewers. Any product that may be evaluated in this article or claim that may be made by its manufacturer is not guaranteed or endorsed by the publisher.

Copyright (๑) $2022 \mathrm{Liu}, \mathrm{Li}$, Zhou and Wiśniewski. This is an open-access article distributed under the terms of the Creative Commons Attribution License (CC BY). The use, distribution or reproduction in other forums is permitted, provided the original author(s) and the copyright owner(s) are credited and that the original publication in this journal is cited, in accordance with accepted academic practice. No use, distribution or reproduction is permitted which does not comply with these terms. 